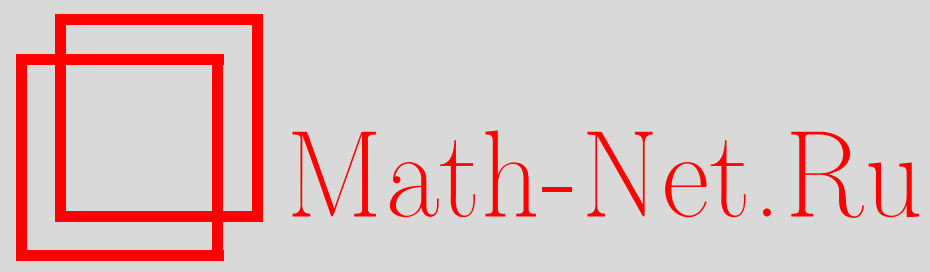

В. М. Храпченко, Принципиальное расхождение между глубиной и задержкой, Дискрет. матем., 2008, том 20, выпуск $3,51-72$

DOI: https://doi.org/10.4213/dm1013

Использование Общероссийского математического портала Math-Net.Ru подразумевает, что вы прочитали и согласны с пользовательским соглашением http://www.mathnet.ru/rus/agreement

Параметры загрузки:

IP : 3.89 .185 .249

26 апреля 2023 г., $16: 48: 17$ 


\title{
Принципиальное расхождение между глубиной и задержкой
}

\author{
() 2008 г. В. М. Храпченко
}

\begin{abstract}
Ранее было показано, что даже у минимальной схемы задержка $T$ может быть значительно меньше глубины $D$. А именно, была построена бесконечная последовательность минимальных схем, у которых $T<\log _{2} D+6$, причем $D \rightarrow \infty$. Этот результат был бы убедительнее, если бы неравенство выполнялось и для всех эквивалентных минимальных схем. В настоящей работе построена бесконечная последовательность булевых функций $F_{k}, k=1,2, \ldots$, такая, что всякая минимальная схема для любой функции $F_{k}$ имеет задержку и глубину, удовлетворяющие неравенству $T<\log _{2} D+14$.

Работа выполнена при поддержке Программы фундаментальных исследований ОПМ РАН «Алгебраические и комбинаторные методы математической кибернетики», проект «Синтез и сложность управляющих систем».
\end{abstract}

Долгое время казалось настолько очевидным, что задержка любой схемы равна ее глубине (в случае, когда задержки элементов равны 1), что не возникало вопроса о проверке этого положения. Однако стоило формализовать понятие задержки (в соответствии с его физическим смыслом), как сразу стало ясно, что по крайней мере в избыточных схемах задержка может быть сколь угодно мала по сравнению с глубиной. Автор показал [1], что почти такое же утверждение справедливо и для заведомо неизбыточных, минимальных схем. В работе [1] было исследовано максимально возможное расхождение между задержкой $T$ и глубиной $D$ для класса минимальных схем с одним выходом над классическим булевым базисом $B_{0}=\left\{\&, \vee,^{-}\right\}$, задержки всех элементов которого равны 1. Была построена бесконечная последовательность минимальных схем, у которых $T<\log _{2} D+6$, причем $D \rightarrow \infty$. С другой стороны, было доказано, что для любой минимальной схемы из рассматриваемого класса выполняется неравенство $T>\log _{2} D$. Тем самым был получен почти окончательный результат.

Однако это не было исчерпывающим решением, поскольку при переходе к другим минимальным схемам, вычисляющим те же функции, что и схемы построенной последовательности, такое большое различие между задержкой и глубиной исчезает. Значит, оставалась возможность истолковать результат как следствие преднамеренного выбора минимальной схемы.

В настоящей работе слегка ослабляется первое неравенство, но зато исключается возможность такого истолкования: построена бесконечная последовательность булевых функций $F_{k}, k=1,2, \ldots$, каждая из которых имеет единственную минимальную схему $S_{k}$ (над базисом $B_{0}$ ) с задержкой $T\left(S_{k}\right)$ и глубиной $D\left(S_{k}\right), D\left(S_{k}\right) \rightarrow \infty$, удовлетворяющими неравенству $T\left(S_{k}\right)<\log _{2} D\left(S_{k}\right)+14$. Формально это можно трактовать даже так: существует бесконечная последовательность булевых функций $F_{k}, k=1,2, \ldots$, такая, 
что всякая минимальная схема (над базисом $B_{0}$ ) для любой функции $F_{k}$ имеет задержку $T$ и глубину $D$, удовлетворяющие неравенству $T<\log _{2} D+14$.

Основные понятия вводятся здесь почти так же, как в [1], но без комментариев. Схемой вычисления (с одним выходом) называется последовательность равенств вида

$$
\begin{aligned}
z_{1} & =\varphi_{1}\left(u_{11}, \ldots, u_{1 r_{1}}\right), \\
& \ldots \\
z_{i} & =\varphi_{i}\left(u_{i 1}, \ldots, u_{i r_{i}}\right), \\
& \ldots \\
z_{l} & =\varphi_{l}\left(u_{l 1}, \ldots, u_{l r_{l}}\right),
\end{aligned}
$$

где каждый символ $u_{i j}, i=1, \ldots, l, j=1, \ldots, r_{i}$, - это либо константа, либо одна из входных переменных $x_{1}, \ldots, x_{n}$, либо одна из внутренних переменных $z_{1}, \ldots, z_{i-1}$, вычисленных на предыдущих шагах, а $\varphi_{1}, \ldots, \varphi_{l}-$ функции (операции) из некоторого заранее заданного множества $B$ (разные символы $u_{i j}$ могут обозначать одну переменную или константу, но $x_{1}, \ldots, x_{n}, z_{1}, \ldots, z_{l}$ - это разные переменные). Выходной переменной является $z_{l}$, кроме вырожденного случая $l=0$, когда это константа либо одна из входных переменных. Множество $B$ называют базисом, над которым построена схема.

Очевидно, что каждая из переменных $z_{1}, \ldots, z_{l}$ может быть выражена через входные переменные $x_{1}, \ldots, x_{n}$, то есть представлена в виде

$$
z_{i}=f_{i}\left(x_{1}, \ldots, x_{n}\right), \quad i=1, \ldots, l .
$$

По определению, схема (1) вычисляет функцию, выражающую выходную переменную через входные, то есть функцию $f_{l}\left(x_{1}, \ldots, x_{n}\right)$, если $l \neq 0$.

Сложность схемы (1) полагается равной $l$, то есть числу операций в ней. Схема (1) называется минимальной (в классе схем над базисом $B$ ), если ее сложность не больше сложности любой схемы над базисом $B$, вычисляющей ту же функцию. Как обычно, сложность схемы $S$ обозначается через $L(S)$, а при заданном базисе $B$ сложность функции $f$ (то есть сложность минимальной схемы, вычисляющей $f$ ) обозначается через $L(f)$.

Схема вычисления графически изображается как схема из функциональных элементов [2], то есть ориентированный ациклический граф (подробнее см. [3]). Каждое понятие вводится для более удобной из этих моделей, а относится к обеим. В случае, когда задержки элементов равны 1 (как в этой статье), глубина схемы $S$ из функциональных элементов определяется как максимальное число элементов в пути этой схемы, идущем от входа к выходу, и обозначается через $D(S)$.

Будем теперь считать, что каждая переменная зависит от времени $t$, пробегающего действительные значения: $x_{j}=x_{j}(t), j=1, \ldots, n ; z_{i}=z_{i}(t), i=1, \ldots, l$. При этом входные переменные $x_{1}, \ldots, x_{n}$ принимают свои значения при $t=0$ и затем не изменяются, то есть при всех $t \geqslant 0$

$$
x_{j}(t)=x_{j}(0)=x_{j}, \quad j=1, \ldots, n,
$$

а значения внутренних переменных $z_{1}, \ldots, z_{l}$ определяются равенствами (1), но с задержкой равной 1 , то есть при всех $t \geqslant 1$

$$
z_{i}(t)=\varphi_{i}\left(u_{i 1}(t-1), \ldots, u_{i r_{i}}(t-1)\right), \quad i=1, \ldots, l .
$$

В начальный промежуток времени $0 \leqslant t<1$ значения внутренних переменных $z_{1}, \ldots, z_{l}$, вообще говоря, могут быть произвольными. 
Задержка схемы (1) определяется (при $l \neq 0$ ) как такое наименьшее число $T$, что какими бы ни были наборы значений внутренних переменных $z_{1}, \ldots, z_{l}$ в промежуток времени $0 \leqslant t<1$ (начальное состояние схемы) и каким бы ни был набор значений входных переменных $x_{1}, \ldots, x_{n}$, при всяком $t \geqslant T$ справедливо равенство $z_{l}(t)=z_{l}(T)$. Такое число существует, так как в силу (2) и (3) при $t \geqslant l$ (независимо от начального состояния схемы) имеет место равенство $z_{l}(t)=f_{l}\left(x_{1}, \ldots, x_{n}\right)$, а точная нижняя грань здесь достигается (см. [4]). Задержка схемы $S$ обозначается через $T(S)$. Ясно, что $T(S) \leqslant D(S)$.

Наконец, надо формализовать понятие единственности схемы. Две схемы вычисления называются изоморфными, если одну из них можно преобразовать в другую перестановкой равенств и соответствующей перенумерацией внутренних переменных (заметим, что не все перестановки допустимы, то есть дают схемы вычисления). Изоморфные схемы вычисления различать не будем. Соответствующие им схемы из функциональных элементов вообще совпадают (если только не помечать выходы элементов внутренними переменными).

Как и в работе [1], рассматриваем схемы над базисом $B_{0}=\left\{\&, \vee,^{-}\right\}$. Ввиду коммутативности операций $\&$ и $\vee$ для схем над базисом $B_{0}$ расширим определение изоморфизма, допуская при преобразовании одной схемы в другую перестановку аргументов этих операций, и снова не будем различать изоморфные схемы.

Функции $F_{k}, k=1,2, \ldots$, зададим, указав вычисляющие их схемы $S_{k}$ (которые в конечном итоге окажутся единственными минимальными схемами для этих функций). Схема $S_{k}$ (рис. 1) состоит из двух частей (детально описанных ниже), причем строение первой части показано прямо на рис. 1 , а строение второй части (блок $\& \vee$ ) показано на рис. 2 .

Положим $m=2^{k}$. Первая часть схемы $S_{k}$ состоит из $m$ микроблоков с номерами $i=1, \ldots, m$, каждый из которых описывается (при своем $i$ ) следующей последовательностью равенств (здесь для удобства многие переменные занумерованы двойными индексами, но их легко перенумеровать по порядку, как в (1)):

$$
\begin{aligned}
z_{i 1}(t) & =z_{i-1,0}(t-1) \& x_{i 1}(t-1), \\
z_{i 2}(t) & =z_{i 1}(t-1) \vee x_{i 2}(t-1), \\
z_{i 3}(t) & =z_{i 2}(t-1) \& x_{i 3}(t-1), \\
z_{i 4}(t) & =z_{i 3}(t-1) \vee x_{i 4}(t-1), \\
z_{i 5}(t) & =z_{i 4}(t-1) \& x_{i 5}(t-1), \\
z_{i 6}(t) & =z_{i 5}(t-1) \vee x_{i 6}(t-1), \\
z_{i 7}(t) & =z_{i 6}(t-1) \& x_{i 7}(t-1), \\
z_{i 8}(t) & =z_{i 7}(t-1) \vee x_{i 8}(t-1), \\
z_{i 9}(t) & =z_{i 8}(t-1) \& x_{i 9}(t-1), \\
z_{i}(t) & =z_{i 9}(t-1) \vee x_{i}(t-1), \\
z_{i 0}(t) & =z_{i 5}(t-1) \vee x_{i 1}(t-1),
\end{aligned}
$$

Первый микроблок отличается только тем, что на левый вход его первого элемента поступает входная переменная $x_{10}$ (а не внутренняя переменная $z_{00}$ ); в последнем $m$-м микроблоке нет элемента, вычисляющего $z_{m 0}$.

Вторая часть схемы $S_{k}$, то есть блок $\& \vee$ (рис. 2), представляет собой $k$-ярусное бинарное дерево, в котором элементы $\&$ и $\vee$ чередуются. Блок $\& \vee$ описывается последова- 


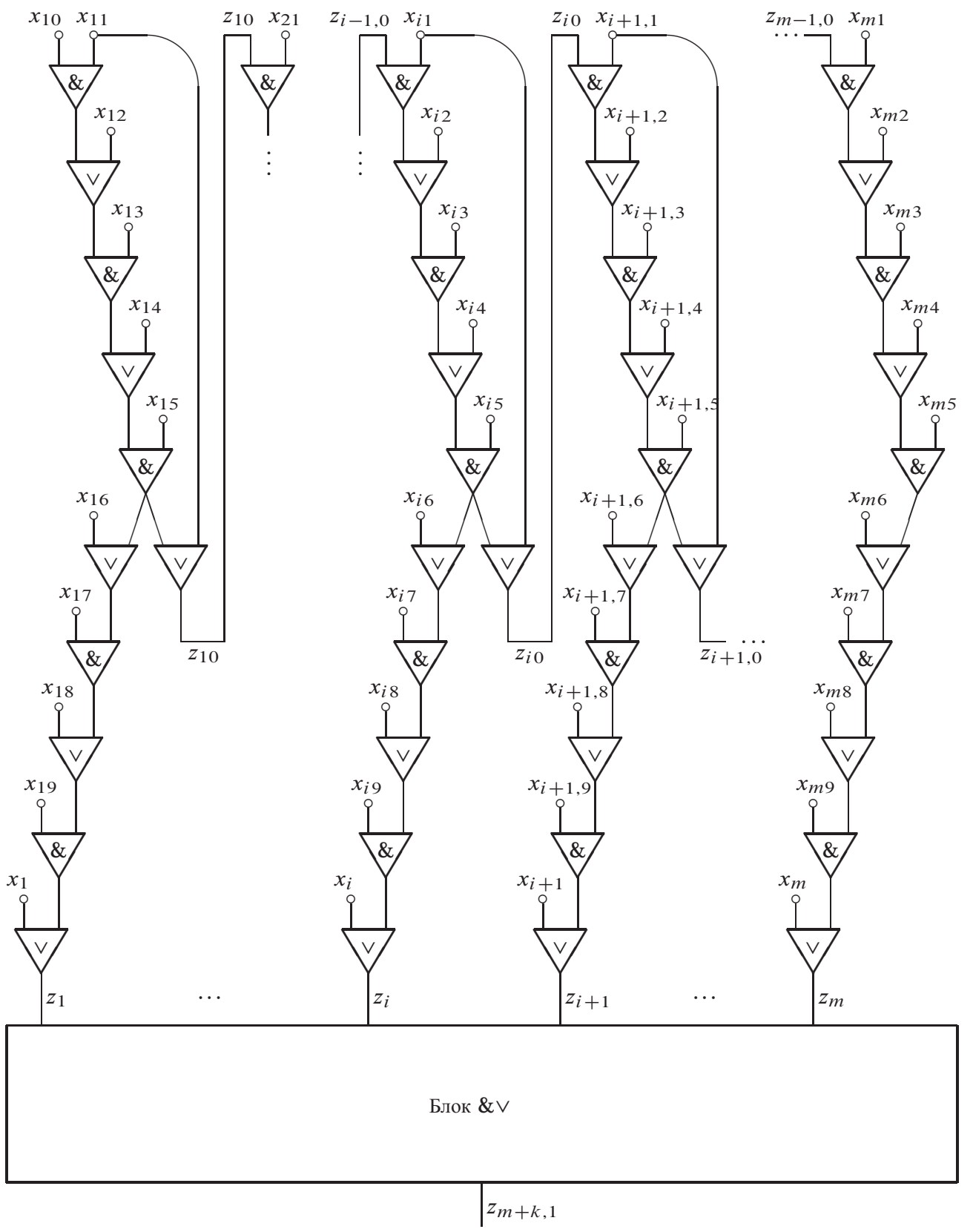

Рис. 1. Схема $S_{k}$ 

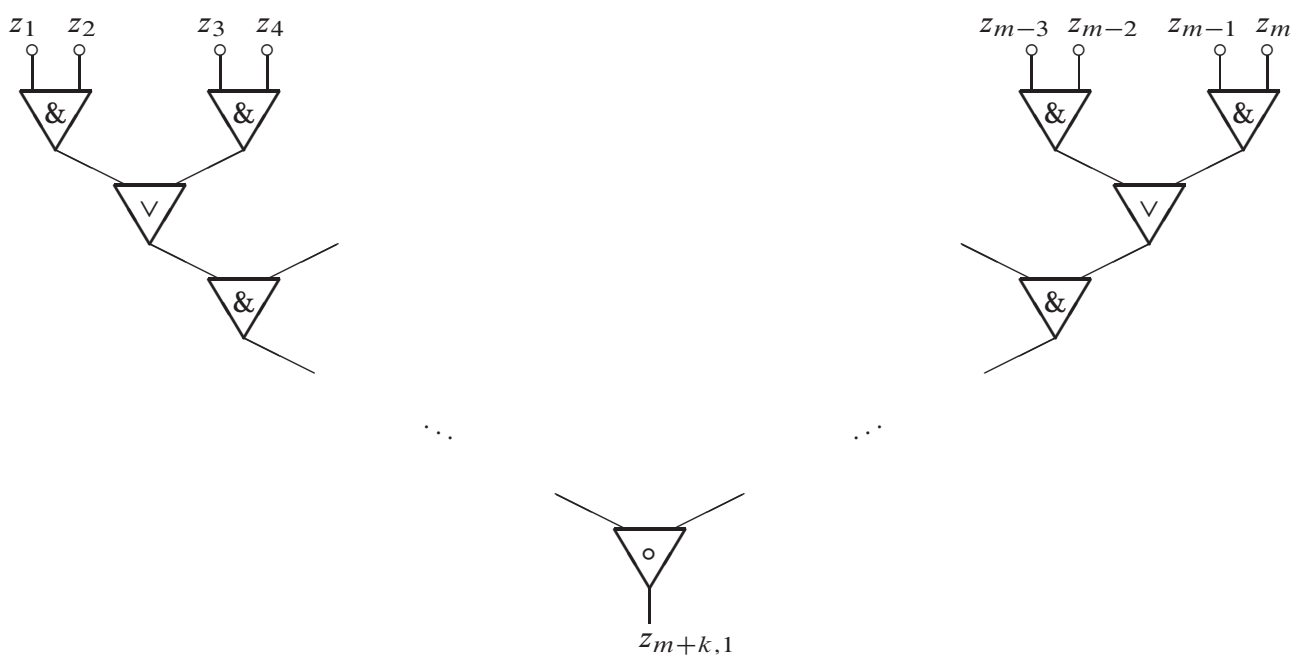

Рис. 2. Блок \&

тельностью равенств

$$
\begin{aligned}
z_{m+1,1}(t) & =z_{1}(t-1) \& z_{2}(t-1), \\
z_{m+1,2}(t) & =z_{3}(t-1) \& z_{4}(t-1), \\
& \cdots \\
z_{m+1,(m / 2)-1}(t) & =z_{m-3}(t-1) \& z_{m-2}(t-1), \\
z_{m+1, m / 2}(t) & =z_{m-1}(t-1) \& z_{m}(t-1), \\
z_{m+2,1}(t) & =z_{m+1,1}(t-1) \vee z_{m+1,2}(t-1), \\
& \cdots \\
z_{m+2, m / 4}(t) & =z_{m+1,(m / 2)-1}(t-1) \vee z_{m+1, m / 2}(t-1), \\
& \cdots \\
z_{m+k, 1}(t) & =z_{m+k-1,1}(t-1) \circ z_{m+k-1,2}(t-1), \quad t \geqslant 1,
\end{aligned}
$$

где операция о есть $\&$, если $k$ нечетно, и $\vee$, если $k$ четно.

Итак, при любом $k, k=1,2, \ldots$, схема $S_{k}$ определена (формально, соотношениями (4) и (5)), а значит определена и вычисляемая ею функция $F_{k}$.

Лемма 1. Глубина и задержка любой схемь $S_{k}, k=1,2, \ldots$, удовлетворяют соотношениям

$$
\begin{aligned}
& D\left(S_{k}\right)=6 \cdot 2^{k}+k+4, \\
& T\left(S_{k}\right) \leqslant k+16,
\end{aligned}
$$


u, как следствие, неравенству

$$
T\left(S_{k}\right)<\log _{2} D\left(S_{k}\right)+14 .
$$

Доказательство. Сопоставляя рис. 1 и 2 , нетрудно увидеть, что глубина $D\left(S_{k}\right)$ достигается на пути, идущем от входа $x_{10}$ к выходу $z_{m+k, 1}$ через $z_{m}$ (этот путь определяется однозначно). В каждом из первых $m-1$ микроблоков верхней (первой) части схемы $S_{k}$ данный путь проходит через 6 элементов, а в $m$-м микроблоке - через 10 элементов. Итого в верхней части он проходит через $6 m+4=6 \cdot 2^{k}+4$ элементов. В блоке $\& \vee$ этот путь проходит еще через $k$ элементов. Отсюда следует утверждение леммы про глубину $D\left(S_{k}\right)$.

Подсчитаем теперь задержку $T\left(S_{k}\right)$. Сначала покажем, что все переменные $z_{i 0}$, $i=1, \ldots, m-1$, вычисляются с задержкой, не большей 6. Вообще говоря, это можно увидеть, воспользовавшись рис. 1: достаточно обратить внимание на то, что как при $x_{i 1}=0$, так и при $x_{i 1}=1, z_{i 0}$ не зависит от $z_{i-1,0}$. Мы придадим этому рассуждению более строгую форму.

Исключая с помощью равенств (4) одну за другой внутренние переменные и учитывая (2), получим, что (знак \& опускаем)

$$
\begin{array}{llrl}
z_{i 0}(t) & =z_{i 5}(t-1) \vee x_{i 1}, & & t \geqslant 1, \\
z_{i 0}(t) & =z_{i 4}(t-2) x_{i 5} \vee x_{i 1}, & & t \geqslant 2, \\
z_{i 0}(t) & =\left(z_{i 3}(t-3) \vee x_{i 4}\right) x_{i 5} \vee x_{i 1}, & t \geqslant 3, \\
z_{i 0}(t) & =\left(z_{i 2}(t-4) x_{i 3} \vee x_{i 4}\right) x_{i 5} \vee x_{i 1}, & t \geqslant 4, \\
z_{i 0}(t)=\left(\left(z_{i 1}(t-5) \vee x_{i 2}\right) x_{i 3} \vee x_{i 4}\right) x_{i 5} \vee x_{i 1}, & & t \geqslant 5, \\
z_{i 0}(t)=\left(\left(z_{i-1,0}(t-6) x_{i 1} \vee x_{i 2}\right) x_{i 3} \vee x_{i 4}\right) x_{i 5} \vee x_{i 1}, & & t \geqslant 6,
\end{array}
$$

где в случае $i=1$ подразумевается, что $z_{00}=x_{10}$. Пользуясь тождеством

$$
\Phi\left(x_{i 1}, \ldots\right) \vee x_{i 1}=\Phi(0, \ldots) \vee x_{i 1},
$$

справедливым для всякой формулы Ф, получим, что

$$
z_{i 0}(t)=\left(x_{i 2} x_{i 3} \vee x_{i 4}\right) x_{i 5} \vee x_{i 1}, \quad t \geqslant 6,
$$

а это и означает, что все переменные $z_{i 0}, i=1, \ldots, m-1$, вычисляются с задержкой, не большей 6 (как, впрочем, и переменная $z_{00}=x_{10}$ ).

Поскольку для вычисления значения переменной $z_{i+1}, i=0,1, \ldots, m-1$, при вычисленном значении переменной $z_{i 0}$ требуются только значения переменных $x_{i+1,1}, \ldots, x_{i+1,9}$ и $x_{i+1}$, переменные $z_{i+1}, i=0,1, \ldots, m-1$, вычисляются с задержкой, не большей 16 (см. рис. 1 или равенства (4)). Точно так же переменная $z_{m+k, 1}$ вычисляется с задержкой $k$ относительно переменных $z_{1}, \ldots, z_{m}$. Отсюда следует, что $T\left(S_{k}\right) \leqslant k+16$.

Тем самым утверждение леммы про задержку $T\left(S_{k}\right)$ тоже доказано. Отметим, что в действительности здесь выполняется равенство, в чем можно было бы убедиться так же, как в [1]. Лемма доказана.

Остается доказать, что схема $S_{k}$ является единственной минимальной схемой (над базисом $B_{0}$ ) для функции $F_{k}$. Время $t$ больше учитывать не надо, и символ $t$ везде далее опускается. Мы будем пользоваться известным приемом - подстановкой константы $(0$ или 1) вместо входной переменной и удалением из схемы элементов, ставших ненужными. Однако при применении этого приема будут две особенности. Во-первых, функция задана не формулой, а схемой (то есть последовательностью равенств), и во-вторых, здесь 
будет недостаточно подсчета числа удаленных элементов - придется также анализировать структуру схем. Это возможно только, когда есть детальное описание всех операций, выполняемых при подстановке константы.

Опишем подстановку константы вместо входной переменной и последующие преобразования схемы вычисления.

Подстановка константы допускается, если выходная переменная не обращается при этом в константу. В рамках выполнения преобразований несущественно расширим базис, добавив тождественную функцию, для которой обозначения не потребуется.

С допустимой подстановкой $x_{j}=\sigma(\sigma-$ константа) связана следующая цепочка преобразований схемы, сохраняющих (начиная со 2-го шага) вычисляемую функцию.

1. В каждом равенстве схемы (содержащем $x_{j}$ ) $x_{j}$ заменяется на $\sigma$.

2. В соответствии с одним из тождеств (с учетом коммутативности)

$$
\begin{array}{lll}
u \& 0=0, & u \vee 0=u, & \overline{0}=1, \\
u \& 1=u, & u \vee 1=1, & \overline{1}=0
\end{array}
$$

правая часть каждого равенства схемы, содержащего константу, заменяется либо на переменную, либо на константу. В итоге каждое такое равенство принимает вид $z_{i}=u$, где $u$ - некоторая переменная или константа.

3. В каждом равенстве схемы, содержащем $z_{i}$ в правой части, $z_{i}$ заменяется на $u$.

4. Из схемы удаляется равенство $z_{i}=u$, полученное на 2-м шаге. Если переменная $z_{i}$ была выходной, то удаляются также все равенства, идущие после равенства, вычисляющего переменную $u$ (все равенства вообще, если $u$ - входная переменная), и $u$ становится выходной переменной.

Если на 2-м шаге получено несколько равенств, то столько же раз повторяются шаги 3 и 4. Если после этого в схеме осталось равенство, содержащее константу, то все шаги, начиная со второго, повторяются.

Описанную здесь процедуру будем называть Подстановкой.

Рассмотрим теперь при каждом $i=1, \ldots, m$ следующую подстановку констант (выполняемую одновременно в функцию $F_{k}$ и в схему $S_{k}$ ):

$$
x_{19}=\cdots=x_{i 9}=x_{i 5}=0 .
$$

В результате этой подстановки в схему $S_{k}$ мы получаем (см. рис. 1 или равенства (4)), что

$$
\begin{aligned}
z_{1} & =x_{1}, \ldots, z_{i}=x_{i}, \\
z_{i 0} & =x_{i 1} .
\end{aligned}
$$

При этом можно удалить не только те элементы, о которых сказано в Подстановке, а вообще все элементы первых $i$ микроблоков, никак не участвующие теперь в вычислении (геометрически, они оказались отрезаны от выхода схемы). Обозначим получившуюся после их удаления схему через $S_{k, m-i}$, имея в виду, что она содержит $m-i$ микроблоков (см. рис. 3).

В частности, схема $S_{k, 0}$ - это блок $\& \vee$, но с входными переменными $x_{1}, \ldots, x_{m}$. Очевидно, что схема $S_{k, m-i}, i=1, \ldots, m$, вычисляет функцию $\left.F_{k}\right|_{x_{19}=\ldots=x_{i 9}=x_{i 5}=0}$ (то есть функцию, получающуюся из $F_{k}$ в результате указанной подстановки), которую мы для сокращения записи обозначим $F_{k, m-i}$. Будем также считать, что $S_{k, m}=S_{k}$ и $F_{k, m}=F_{k}$.

Нашей целью является доказать, что при любом $m-i, m-i=0,1, \ldots, m$, схема $S_{k, m-i}$ является единственной минимальной схемой (над базисом $B_{0}$ ) для функции $F_{k, m-i}$. 


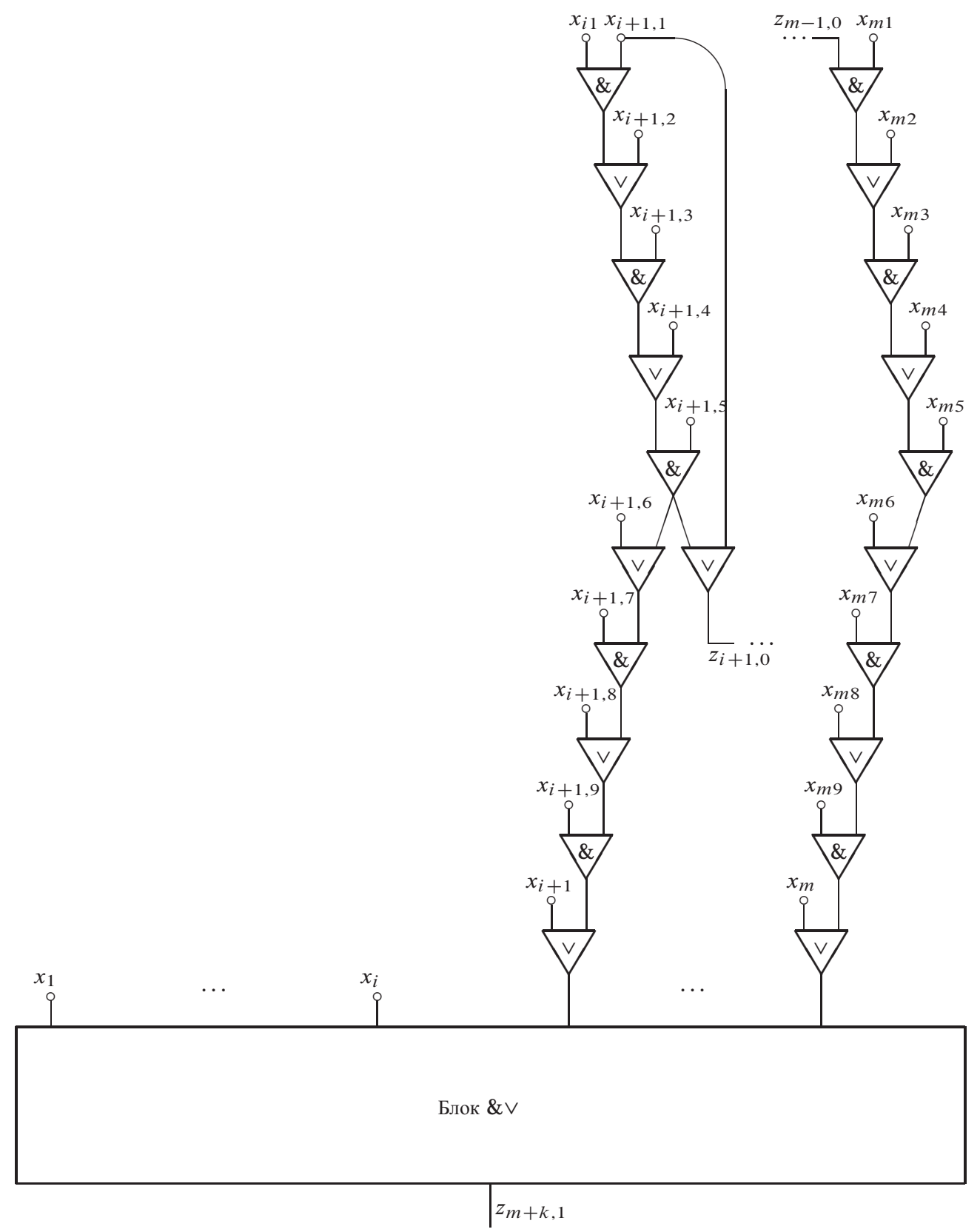

Рис. 3. Схема $S_{k, m-i}$ 
Лемма 2. Пусть схема $S$ является единственной минимальной схемой над базисом $B_{0}$ для булевой функции $f\left(x_{1}, \ldots, x_{n}\right)$ и переменная $x_{1}$ входит только в равенства с опе-

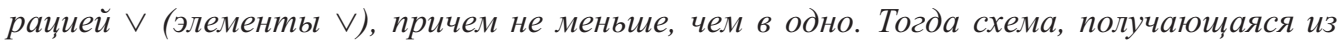
схемы $S$ в результате замены всех вхождений переменной $x_{1}$ новой переменной $z_{i_{0}} u$ добавления равенства $z_{i_{0}}=x_{0} \& x_{1}$ (замень переменной $x_{1}$ элементом $x_{0} \& x_{1}$ ) с новой переменной $x_{0}$, является единственной минимальной схемой над базисом $B_{0}$ для функции $f\left(x_{0} \& x_{1}, x_{2}, \ldots, x_{n}\right)$.

Доказательство. Пусть $f=f\left(x_{1}, x_{2}, \ldots, x_{n}\right), f_{0}=f\left(x_{0} \& x_{1}, x_{2}, \ldots, x_{n}\right)$.

Переменная $x_{1}$ является существенной переменной функции $f$, так как иначе, подставив в схему $S$ вместо $x_{1}$ константу 0 и сделав упрощения вида $0 \vee u=u$, мы получили бы схему над базисом $B_{0}$ меньшей сложности, вычисляющую ту же функцию $f$, а это противоречит минимальности схемы $S$. Отсюда сразу следует, что, во-первых, функция $f$ не является тождественной константой, а во-вторых, переменные $x_{0}$ и $x_{1}$ являются существенными для функции $f_{0}$.

Рассмотрим произвольную минимальную схему (над базисом $B_{0}$ ) для функции $f_{0}$. Обозначим ее через $S_{0}$. Сделаем в ней подстановку $x_{0}=1$ и удалим все равенства (элементы), в которые входит $x_{0}$, а также, быть может, некоторые еще (см. Подстановку). В результате получится некоторая схема $S^{\prime}$, вычисляющая функцию $f$. Так что подстановка была допустимой. Пусть $a-$ число удаленных равенств (элементов). Тогда

$$
L\left(f_{0}\right)=L\left(S_{0}\right)=L\left(S^{\prime}\right)+a \geqslant L(f)+a .
$$

В силу существенной зависимости функции $f_{0}$ от переменной $x_{0}$, эта переменная входит хотя бы в одно равенство (один элемент) схемы $S_{0}$. Следовательно, $a \geqslant 1$. С другой стороны, из определения функции $f_{0}$ вытекает, что

$$
L\left(f_{0}\right) \leqslant L(f)+1 .
$$

Из полученных соотношений следует, в частности, что

$$
\begin{aligned}
a & =1, \\
L\left(S^{\prime}\right) & =L(f) .
\end{aligned}
$$

В силу (6) переменная $x_{0}$ входит только в одно равенство (элемент) схемы $S_{0}$. Вычисляемая им переменная, очевидно, тоже входит в какое-то равенство (элемент) схемы $S_{0}$ или является выходной. Но тогда при подстановке $x_{0}=1$ она не должна обращаться в константу, так как это вело бы либо к удалению еще хотя бы одного равенства (элемента) либо к противоречию с допустимостью этой подстановки. Поэтому единственное равенство схемы $S_{0}$, содержащее переменную $x_{0}$, не может иметь операцией ни $\vee$, ни ${ }^{-}$, а значит (с точностью до коммутативности) оно имеет вид

$$
z_{i_{0}}=x_{0} \& u_{i_{0}}
$$

где $z_{i_{0}}$ и $u_{i_{0}}-$ некоторые переменные.

Аналогичным образом устанавливаем, что переменная $x_{1}$ входит только в одно равенство схемы $S_{0}$, которое (с точностью до коммутативности) имеет вид

$$
z_{i_{1}}=x_{1} \& u_{i_{1}}
$$

где $z_{i_{1}}$ и $u_{i_{1}}-$ некоторые переменные. 
Ввиду (7) схема $S^{\prime}$ является минимальной схемой для функции $f$ и, стало быть, совпадает со схемой $S$, причем из сказанного выше следует, что схема $S=S^{\prime}$ получается из схемы $S_{0}$ в результате удаления одного равенства (элемента), а именно, равенства (8).

Далее, согласно условию леммы схема $S$ не может содержать равенства вида (9), которое есть в схеме $S_{0}$. Это значит, что при подстановке $x_{0}=1$ равенство (9) удаляется, но тогда оно совпадает с равенством (8). Поэтому $z_{i_{0}}=z_{i_{1}}, u_{i_{0}}=x_{1}, u_{i_{1}}=x_{0}$, и единое равенство имеет вид

$$
z_{i_{0}}=x_{0} \& x_{1}
$$

причем обе переменные $x_{0}$ и $x_{1}$ входят только в это равенство схемы $S_{0}$.

Итак, в схеме $S_{0}$ по сравнению со схемой $S$ есть дополнительно только одно равенство (10). Чтобы выяснить, куда поступает вычисляемая им переменная $z_{i_{0}}$, надо для подстановки $x_{0}=1$ в схему $S_{0}$ проделать все шаги Подстановки, а затем повторить их в обратном порядке.

Шаги Подстановки будут следующие.

1. Равенство (10) заменяется равенством $z_{i_{0}}=1 \& x_{1}$.

2. Последнее равенство заменяется равенством $z_{i_{0}}=x_{1}$.

3. Во всех остальных равенствах $z_{i_{0}}$ заменяется на $x_{1}$.

4. Удаляется равенство $z_{i_{0}}=x_{1}$.

В итоге получаем схему $S$, причем выходная переменная не изменяется, так как переменная $x_{1}$ не может быть выходной в схеме $S$.

Двигаясь в обратную сторону, добавляем к схеме $S$ равенство $z_{i_{0}}=x_{1}$. Далее, некоторые (может быть, все) вхождения переменной $x_{1}$ в схему $S$ заменяем на $z_{i_{0}}$. Наконец, заменяем равенство $z_{i_{0}}=x_{1}$ сначала равенством из шага 1 , а затем равенством (10). При правильном выборе вхождений переменной $x_{1}$, подлежащих замене, получается схема $S_{0}$.

Такой выбор делается почти тривиально. Если бы какое-то вхождение переменной $x_{1}$ в схему $S$ не заменялось на $z_{i_{0}}$, то переменная $x_{1}$ входила бы по крайней мере в два равенства схемы $S_{0}$ (в равенство (10) и в то равенство схемы $S$, в котором не было сделано замены), что противоречит доказанному выше. Следовательно, при переходе от схемы $S$ к схеме $S_{0}$ все вхождения переменной $x_{1}$ заменяются на $z_{i_{0}}$.

Итак, оказалось, что произвольная минимальная схема $S_{0}$ для функции $f_{0}$ совпадает со схемой, описанной в утверждении леммы. Это значит, что минимальная схема у функции $f_{0}$ единственна и получается она так, как об этом сказано в лемме.

Из леммы 2 в силу принципа двойственности вытекает следующее утверждение.

Лемма 3. Пусть схема $S$ является единственной минимальной схемой над базисом $B_{0}$ для булевой функции $f\left(x_{1}, \ldots, x_{n}\right)$ и переменная $x_{1}$ входит только в равенства с операцией \& (элементы \&), причем не меньше, чем в одно. Тогда схема, получаюшаяся из схемы $S$ в результате замены всех вхождений переменной $x_{1}$ новой переменной $z_{i_{0}} u$ добавления равенства $z_{i_{0}}=x_{0} \vee x_{1}$ (замень переменной $x_{1}$ элементом $x_{0} \vee x_{1}$ ) с новой переменной $x_{0}$, является единственной минимальной схемой над базисом $B_{0}$ для функции $f\left(x_{0} \vee x_{1}, x_{2}, \ldots, x_{n}\right)$.

Лемма 4. Схема $S_{k}=S_{k, m}$ является единственной минимальной схемой (над базисом $\left.B_{0}\right)$ для функиии $F_{k}=F_{k, m}$. 
Доказательство. Индукцией по $m-i$, начиная с $m-i=0$ и кончая $m-i=m$, докажем, что каждая схема $S_{k, m-i}$ является единственной минимальной схемой (над базисом $B_{0}$ ) для функции $F_{k, m-i}$.

Начальный шаг индукиии: $m-i=0(i=m)$. Схема $S_{k, 0}$ (как уже отмечалось выше) это фактически блок $\& \vee$ (см. рис. 2) только с входными переменными $x_{1}, \ldots, x_{m}$. Схему $S_{k, 0}$ можно построить, например, так: взять ее выходной элемент и добавлять по очереди ярусы один за другим, а в пределах яруса на каждом шаге добавлять по одному элементу, двигаясь слева направо. Переменные $x_{1}, \ldots, x_{m}$ можно вводить в любом порядке. Опишем этот процесс более формально, выбрав удобный порядок введения переменных и считая для определенности, что $k$ четное, а значит, о $=\vee$.

Исходная схема:

1-й шаг.

2-й шаг.

3-й шаг.

6-й шаг.

7-й шаг.

$(m-2)$-й шаг. $z_{m+k, 1}=x_{m / 2} \vee x_{m}$.

Замена $x_{m / 2}$ на $z_{m+k-1,1}=x_{m / 4} \& x_{m / 2}$.

Замена $x_{m}$ на $z_{m+k-1,2}=x_{3 m / 4} \& x_{m}$.

Замена $x_{m / 4}$ на $z_{m+k-2,1}=x_{m / 8} \vee x_{m / 4}$.

Замена $x_{m}$ на $z_{m+k-2,4}=x_{7 m / 8} \vee x_{m}$.

Замена $x_{m / 8}$ на $z_{m+k-3,1}=x_{m / 16} \& x_{m / 8}$.

Замена $x_{m}$ на $z_{m+1, m / 2}=x_{m-1} \& x_{m}$.

Очевидно, что исходная схема является единственной минимальной схемой для функции $x_{m / 2} \vee x_{m}$. Применяя на каждом шаге либо лемму 2, либо лемму 3 , убеждаемся, что полученная после $(m-2)$-го шага схема $S_{k, 0}$ является единственной минимальной схемой для функции $F_{k, 0}$. Точно так же рассматривается случай нечетного $k$.

Индуктивный переход. Пусть уже доказано, что схема $S_{k, m-i}$ (см. рис. 3) является единственной минимальной схемой для функции $F_{k, m-i}$. Докажем аналогичное утверждение для схемы $S_{k, m-i+1}$.

Заметим, что схема $S_{k, m-i+1}$ получается из схемы $S_{k, m-i}$ путем добавления $i$-го микроблока и подачи переменной $z_{i}$ на тот вход, где была переменная $x_{i}$, и переменной $z_{i 0}$ на тот вход, где была переменная $x_{i 1}$ (рис. 4).

Случай $i=m$ отличается тем, что в схеме $S_{k, 1}$ нет элемента, вычисляющего переменную $z_{i 0}=z_{m 0}$. Поэтому в случае $m-i=0$ для доказательства достаточно просто несколько раз применить по очереди леммы 2 и 3.

Общий случай немного сложнее. Пусть $m-i \geqslant 1$. В схему $S_{k, m-i+1}$ (см. рис. 4 ) подставим $x_{i 4}=1$ и после удаления элементов с входами $x_{i-1,1}, x_{i 2}, x_{i 3}, x_{i 4}, x_{i 5}$ получим схему, в которой от $i$-го микроблока останется 6 элементов. Обозначим эту схему через $S_{k, m-i}^{6}$ (рис. 5), а вычисляемую ею функцию через $F_{k, m-i}^{6}$.

На первом этапе будет доказано, что схема $S_{k, m-i}^{6}$ является единственной минимальной схемой для функции $F_{k, m-i}^{6}$.

Сначала проделаем построения в соответствии с леммами 2 и 3 . В схеме $S_{k, m-i}$ (см. рис. 3) заменим $x_{i 1}$ на $z_{i 0}=x_{i 5} \vee x_{i 1}$. Полученную схему обозначим через $S_{k, m-i}^{1}$ (см. рис. 6, где эта схема выделена пунктиром).

По лемме 3 , она является единственной минимальной схемой для вычисляемой ею функции $F_{k, m-i}^{1}$. Кроме того, она содержит на 5 элементов меньше, чем схема $S_{k, m-i}^{6}$ (см. рис. 5 и 6). Поэтому

$$
L\left(F_{k, m-i}^{6}\right) \leqslant L\left(S_{k, m-i}^{6}\right)=L\left(S_{k, m-i}^{1}\right)+5=L\left(F_{k, m-i}^{1}\right)+5 .
$$

Отметим, что в схеме $S_{k, m-i}^{1}$ переменная $x_{i}$ поступает на вход элемента $\&$ (см. рис. 3 и 2) и заменим ее на $z_{i}=x_{i} \vee x_{i 9}$, далее заменим $x_{i 9}$ на $z_{i 9}=x_{i 9} \& x_{i 8}$, и наконец, $x_{i 8}$ 


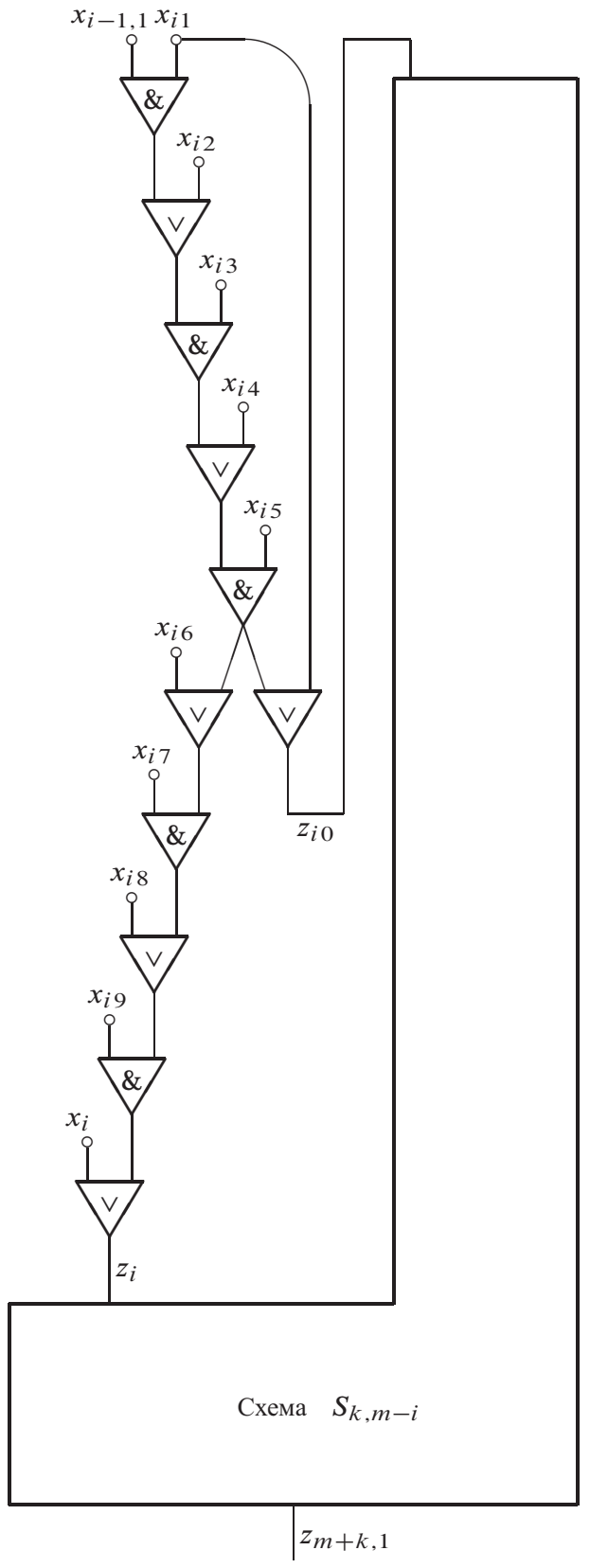

Рис. 4. Схема $S_{k, m-i+1}$ при $i<m$ 


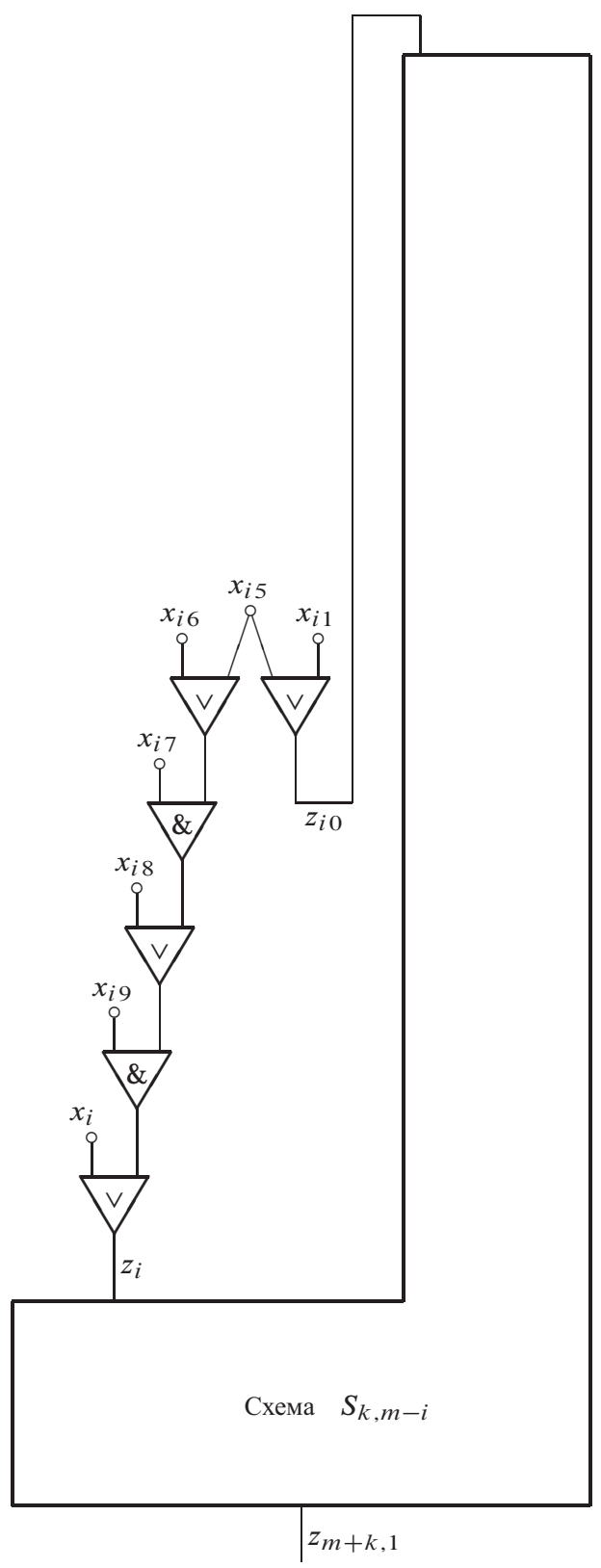

Рис. 5. Схема $S_{k, m-i}^{6}$ при $i<m$ 


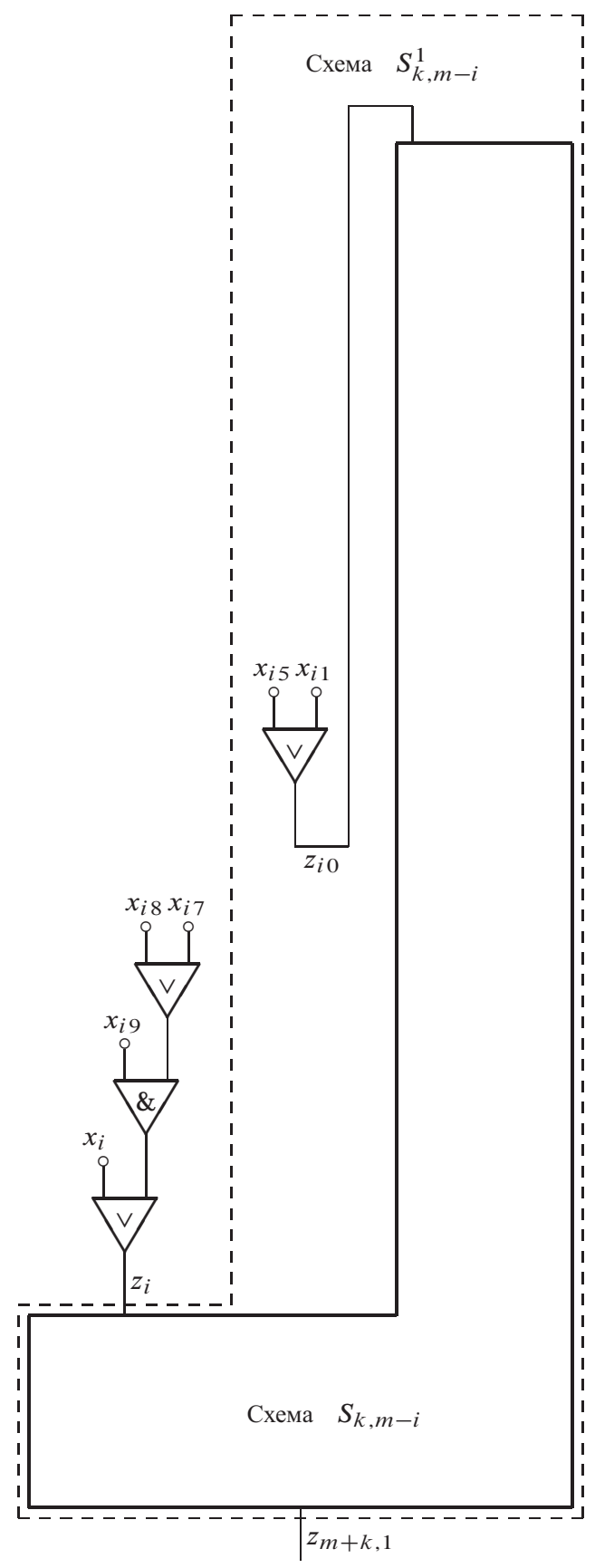

Рис. 6. Схема $S_{k, m-i}^{4}$ при $i<m$ 
на $z_{i 8}=x_{i 8} \vee x_{i 7}$. Последнюю из полученных схем обозначим через $S_{k, m-i}^{4}$ (рис. 6). В силу лемм 2 и 3 она является единственной минимальной схемой для вычисляемой ею функции $F_{k, m-i}^{4}$.

Пусть теперь $S$ - произвольная минимальная схема, вычисляющая функцию $F_{k, m-i}^{6}$. Мы покажем, что схема $S$ имеет в точности такое же строение, как и схема $S_{k, m-i}^{6}$, или, иными словами, совпадает с ней.

Сначала докажем, что в схеме $S$ переменная $x_{i 6}$ поступает на вход только одного элемента и этот элемент есть элемент $\vee$. С этой целью в схему $S$ подставим $x_{i 6}=0$ и удалим элементы (равенства) в соответствии с Подстановкой. Полученную схему обозначим через $S^{\prime}$, а число удаленных элементов (равенств) - через $a^{\prime}$. Прежде всего покажем, что

$$
a^{\prime} \geqslant 1
$$

Поскольку аналогичные неравенства встретятся еще не раз, изложим способ рассуждения поподробнее. Чтобы исследовать функцию $F_{k, m-i}^{6}$, вычисляемую схемой $S$, рассматривается схема $S_{k, m-i}^{6}$ (рис. 5), вычисляющая ту же функцию. В нее делаются подстановки $x_{i 5}=0, x_{i 7}=1, x_{i 8}=0, x_{i 9}=1, x_{i}=0$ и $x_{i+1,9}=\ldots=x_{m 9}=0$, после чего она превращается в схему $S_{k, 0}$, то есть в блок $\& \vee$ (см. рис. 2 ) с тем лишь отличием, что место переменной $z_{i}$ заняла переменная $x_{i}$. В силу минимальности этой схемы ее выход $z_{m+k, 1}$ существенно зависит от всех входных переменных, в том числе и от переменной $x_{i 6}$. Но тогда и выход схемы $S_{k, m-i}^{6}$, а иными словами, функция $F_{k, m-i}^{6}$ также существенно зависит от переменной $x_{i 6}$. Поэтому в схеме $S$, вычисляющей эту же функцию, переменная $x_{i 6}$ поступает на вход хотя бы одного ее элемента. Отсюда следует неравенство (12).

В схему $S^{\prime}$ подставим $x_{i 7}=1$ и удалим элементы (равенства) в соответствии с Подстановкой. Обозначим получившуюся схему через $\hat{S}^{\prime}$, а вычисляемую ею функцию через $\hat{F}^{\prime}$. Так же, как и выше, доказывается, что при переходе к схеме $\widehat{S}^{\prime}$ по крайней мере один элемент (одно равенство) удаляется. Подводя итог после выполнения двух подстановок, мы можем написать

$$
L\left(F_{k, m-i}^{6}\right)=L(S) \geqslant a^{\prime}+1+L\left(\hat{S}^{\prime}\right) \geqslant a^{\prime}+1+L\left(\hat{F}^{\prime}\right) .
$$

Пусть теперь $\hat{S}_{\min }^{\prime}$ - какая-нибудь минимальная схема, вычисляющая функцию $\hat{F}^{\prime}$. Как и выше, доказывается, что в схеме $\widehat{S}_{\min }^{\prime}$ переменная $x_{i 8}$ поступает на вход хотя бы одного элемента. Если это элемент $\&$ или элемент ${ }^{-}$, то в схему $\hat{S}_{\min }^{\prime}$ подставим $x_{i 8}=0$, а если это элемент $\vee$, то подставим $x_{i 8}=1$. В любом случае удаляется по крайней мере два элемента и получается схема, в которой переменная $x_{i}$ поступает на вход хотя бы одного элемента (при доказательстве оба случая приходится рассматривать отдельно). Подставив в полученную схему $x_{i 9}=0$ и удалив по крайней мере один элемент, в обоих случаях получим схему, вычисляющую ту же функцию, что и схема $S_{k, m-i}^{1}$, то есть функцию $F_{k, m-i}^{1}$. Чтобы убедиться в этом, надо в схему $S_{k, m-i}^{6}$ (рис. 5), вычисляющую ту же функцию $F_{k, m-i}^{6}$, что и схема $S$, сделать те же подстановки $x_{i 6}=0, x_{i 7}=1, x_{i 8}=0$ или $x_{i 8}=1, x_{i 9}=0$ и заметить, что при этом получается схема $S_{k, m-i}^{1}$ (см. рис. 6). Подводя итог после выполнения двух очередных подстановок, мы можем написать

$$
L\left(\hat{F}^{\prime}\right) \geqslant 3+L\left(F_{k, m-i}^{1}\right) .
$$

Из (13) и (14) получаем, что

$$
L\left(F_{k, m-i}^{6}\right) \geqslant a^{\prime}+4+L\left(F_{k, m-i}^{1}\right),
$$

3 Дискретная математика, т.20 №3 
откуда ввиду (11) следует, что $a^{\prime} \leqslant 1$, и, стало быть (см. (12)), $a^{\prime}=1$.

Это означает, что в схеме $S$ переменная $x_{i 6}$ поступает на вход только одного элемента, причем он не может быть ни элементом \&, ни элементом - (в этих случаях удалялось бы не менее двух элементов), то есть это элемент $\vee$. Соответствующее равенство имеет вид

$$
z_{i 6}^{\prime}=x_{i 6} \vee u_{i 6},
$$

где $z_{i 6}^{\prime}$ и $u_{i 6}-$ некоторые переменные.

Покажем теперь, что в схеме $S$ переменная $x_{i 7}$ также поступает на вход только одного элемента, но в данном случае этот элемент есть элемент $\&$. В схему $S$ подставим $x_{i 7}=1$ и удалим элементы (равенства) в соответствии с Подстановкой. Полученную схему обозначим через $S^{\prime \prime}$, а число удаленных элементов (равенств) через $a^{\prime \prime}$. Как и выше, доказывается, что $a^{\prime \prime} \geqslant 1$. В схему $S^{\prime \prime}$ подставим $x_{i 6}=0$ и удалим элементы (равенства) в соответствии с Подстановкой. Обозначим получившуюся схему через $\widehat{S}^{\prime \prime}$, а вычисляемую ею функцию через $\hat{F}^{\prime \prime}$. Так же, как и выше, доказывается, что при переходе к схеме $\widehat{S}^{\prime \prime}$ удаляется по крайней мере один элемент (одно равенство). Поэтому

$$
L\left(F_{k, m-i}^{6}\right) \geqslant a^{\prime \prime}+1+L\left(\hat{F}^{\prime \prime}\right) .
$$

Замечая, что $\hat{F}^{\prime}=\hat{F}^{\prime \prime}$, и пользуясь (11) и (14), приходим к неравенству $a^{\prime \prime} \leqslant 1$ и, как следствие, к равенству $a^{\prime \prime}=1$. Значит, в схеме $S$ переменная $x_{i 7}$ поступает на вход только одного элемента, причем он не может быть ни элементом $\vee$, ни элементом ${ }^{-}$, то есть это элемент $\&$. Соответствующее равенство имеет вид

$$
z_{i 7}^{\prime}=x_{i 7} \& u_{i 7},
$$

где $z_{i 7}^{\prime}$ и $u_{i 7}-$ некоторые переменные.

Итак, схема $S$ содержит равенства (15) и (16), причем знаки операций показывают, что это разные равенства. Подставив в нее $x_{i 6}=1$ и удалив по крайней мере два равенства (равенство (15) и его преемника, то есть равенство, содержащее переменную $z_{i 6}^{\prime}$ в правой части), мы получим схему, вычисляющую ту же функцию $F_{k, m-i}^{4}$, что и схема $S_{k, m-i}^{4}$ (аналогичное рассуждение уже было - для доказательства надо в схему $S_{k, m-i}^{6}$ (рис.5) подставить $\left.x_{i 6}=1\right)$. Полученная схема содержит по крайней мере на два равенства меньше, чем схема $S_{k, m-i}^{6}$, и следовательно, не больше равенств, чем схема $S_{k, m-i}^{4}$. Но тогда она минимальна (для функции $F_{k, m-i}^{4}$ ), а значит, по доказанному совпадает со схемой $S_{k, m-i}^{4}$.

Теперь уже ясно, что схема $S$ получается добавлением к схеме $S_{k, m-i}^{4}$ равенства (15) и его преемника в схеме $S$ (никакие другие равенства при подстановке $x_{i 6}=1$ не удаляются - это противоречило бы минимальности схемы $\left.S_{k, m-i}^{4}\right)$. Далее, в схеме $S_{k, m-i}^{4}$ переменная $x_{i 7}$ не поступает на вход элемента $\&$ (см. рис. 6). Это значит, что при подстановке $x_{i 6}=1$ равенство (16) удаляется, то есть именно оно является преемником равенства (15) в схеме $S$. Отсюда вытекает, что $u_{i 7}=z_{i 6}^{\prime}$ и что при переходе от схемы $S$ к схеме $S_{k, m-i}^{4}$ переменная $z_{i 7}^{\prime}$ заменяется переменной $x_{i 7}$. Таким образом, равенство (16) принимает окончательный вид

$$
z_{i 7}^{\prime}=x_{i 7} \& z_{i 6}^{\prime},
$$

где $z_{i 6}^{\prime}$ определяется равенством (15), и известно, что при обратном переходе от схемы $S_{k, m-i}^{4}$ к схеме $S$ переменная $x_{i 7}$ (см. рис.6) заменяется переменной $z_{i 7}^{\prime}$. 
Покажем теперь, что $u_{i 6}=x_{i 5}$. Действительно, в противном случае переменная $x_{i 5}$ в схеме $S$ поступала бы на вход только одного элемента (это видно из сопоставления рис. 6 с равенствами (15) и (17)). Но тогда при подстановке $x_{i 1}=1$ в схему $S$ пропадала бы существенная зависимость выхода схемы от переменной $x_{i 5}$, а это противоречит тому, что при этой же подстановке в схему $S_{k, m-i}^{6}$, вычисляющую ту же функцию, существенная зависимость выхода схемы от переменной $x_{i 5}$ сохраняется. Следовательно, $u_{i 6}=x_{i 5}$ и равенство (15) принимает окончательный вид

$$
z_{i 6}^{\prime}=x_{i 6} \vee x_{i 5}
$$

Добавляя к схеме $S_{k, m-i}^{4}$ (рис. 6) равенства (17) и (18) и заменяя в ней $x_{i 7}$ на $z_{i 7}^{\prime}$, получаем схему $S$. Таким образом, оказалось, что априори произвольная минимальная схема $S$ для функции $F_{k, m-i}^{6}$ определяется однозначно и совпадает со схемой $S_{k, m-i}^{6}$. Следовательно, схема $S_{k, m-i}^{6}$ является единственной минимальной схемой для функции $F_{k, m-i}^{6}$.

На втором этапе будет доказано, что схема $S_{k, m-i+1}$ (рис.4) является единственной минимальной схемой для функции $F_{k, m-i+1}$, и индуктивный переход будет завершен. Второй этап полностью аналогичен первому, поэтому многие пояснения опускаются.

Исходя из доказанного на первом этапе, выполним следующие построения. В схеме $S_{k, m-i}^{6}$ (рис. 5) заменим $x_{i 5}$ на $z_{i 5}=x_{i 4} \& x_{i 5}$, затем заменим $x_{i 4}$ на $z_{i 4}=x_{i 3} \vee x_{i 4}$ и, наконец, $x_{i 3}$ на $z_{i 3}=x_{i 2} \& x_{i 3}$. Полученную схему обозначим через $S_{k, m-i}^{9}$ (рис. 7 ).

В силу лемм 2 и 3 она является единственной минимальной схемой для вычисляемой ею функции $F_{k, m-i}^{9}$.

Пусть теперь $R$ - произвольная минимальная схема, вычисляющая функцию $F_{k, m-i+1}$. В схему $R$ подставим $x_{i-1,1}=1$ и удалим элементы (равенства) в соответствии с Подстановкой. Полученную схему обозначим через $R^{\prime}$, а число удаленных элементов (равенств) - через $b^{\prime}$. Как и прежде, доказывается, что $b^{\prime} \geqslant 1$. В схему $R^{\prime}$ подставим $x_{i 2}=0$ и удалим еще по крайней мере один элемент. Обозначим получившуюся схему через $\hat{R}^{\prime}$, а вычисляемую ею функцию через $\hat{G}^{\prime}$. В итоге после двух подстановок мы можем написать

$$
L\left(F_{k, m-i+1}\right) \geqslant b^{\prime}+1+L\left(\hat{G}^{\prime}\right) .
$$

Пусть теперь $\hat{R}_{\min }^{\prime}$ - какая-нибудь минимальная схема, вычисляющая функцию $\hat{G}^{\prime}$. В этой схеме переменная $x_{i 3}$ поступает на вход хотя бы одного элемента. Если это элемент $\&$ или элемент ${ }^{-}$, то в схему $\hat{R}_{\text {min }}^{\prime}$ подставим $x_{i 3}=0$, а если это элемент $\vee$, то подставим $x_{i 3}=1$. В любом случае удаляется по крайней мере два элемента и получается схема, в которой переменная $x_{i 4}$ поступает на вход хотя бы одного элемента (два случая). Подставив в эту схему $x_{i 4}=1$ и удалив еще по крайней мере один элемент, в обоих случаях получим схему, вычисляющую ту же функцию, что и схема $S_{k, m-i}^{6}$ (рис.5), то есть функцию $F_{k, m-i}^{6}$. Чтобы убедиться в этом, надо делать те же подстановки в схему $S_{k, m-i+1}$ (рис. 4). В итоге после двух очередных подстановок мы можем написать

$$
L\left(\hat{G}^{\prime}\right) \geqslant 3+L\left(F_{k, m-i}^{6}\right) .
$$

Из (19) и (20) получаем, что

$$
L\left(F_{k, m-i+1}\right) \geqslant b^{\prime}+4+L\left(F_{k, m-i}^{6}\right),
$$

а отсюда в силу соотношений

$$
L\left(F_{k, m-i+1}\right) \leqslant L\left(S_{k, m-i+1}\right)=L\left(S_{k, m-i}^{6}\right)+5=L\left(F_{k, m-i}^{6}\right)+5
$$




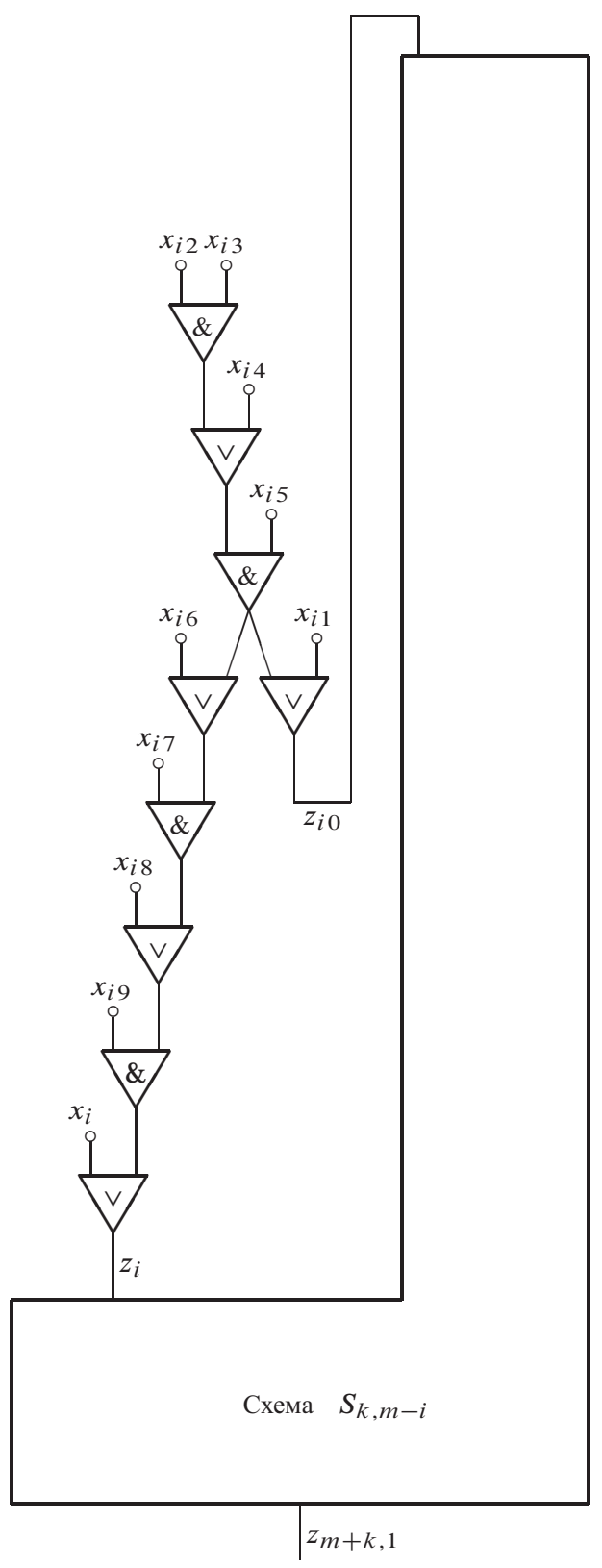

Рис. 7. Схема $S_{k, m-i}^{9}$ при $i<m$ 
(ср. рис. 4 и 5) следует, что $b^{\prime} \leqslant 1$, и стало быть, $b^{\prime}=1$.

Это означает, что в схеме $R$ переменная $x_{i-1,1}$ поступает на вход только одного элемента, причем это элемент $\&$. Соответствующее равенство имеет вид

$$
z_{i 1}^{\prime}=x_{i-1,1} \& u_{i 1}
$$

где $z_{i 1}^{\prime}$ и $u_{i 1}-$ некоторые переменные.

Теперь в схему $R$ подставим $x_{i 2}=0$ и обозначим число удаленных элементов через $b^{\prime \prime}\left(b^{\prime \prime} \geqslant 1\right)$. Подставив в полученную схему $x_{i-1,1}=1$ и удалив еще по крайней мере один элемент, придем к схеме, вычисляющей функцию $\widehat{G}^{\prime}$. Поэтому

$$
L\left(F_{k, m-i+1}\right) \geqslant b^{\prime \prime}+1+L\left(\widehat{G}^{\prime}\right),
$$

откуда, как и выше, следует, что $b^{\prime \prime}=1$. Значит, в схеме $R$ переменная $x_{i 2}$ поступает на вход только одного элемента, причем это элемент $\vee$. Соответствующее равенство имеет вид

$$
z_{i 2}^{\prime}=u_{i 2} \vee x_{i 2},
$$

где $z_{i 2}^{\prime}$ и $u_{i 2}-$ некоторые переменные.

Итак, схема $R$ содержит равенства (21) и (22). Подставив в нее $x_{i-1,1}=0$ и удалив по крайней мере два равенства (равенство (21) и его преемника, то есть равенство, содержащее переменную $z_{i 1}^{\prime}$ в правой части), мы получим схему, вычисляющую ту же функцию $F_{k, m-i}^{9}$, что и схема $S_{k, m-i}^{9}$ (cp. рис. 4 и 7). Эта схема содержит по крайней мере на два равенства меньше, чем схема $S_{k, m-i+1}$, и следовательно, не больше равенств, чем схема $S_{k, m-i}^{9}$. Но тогда она минимальна (для функции $F_{k, m-i}^{9}$ ), а значит, по доказанному совпадает со схемой $S_{k, m-i}^{9}$.

Теперь уже ясно, что схема $R$ получается добавлением к схеме $S_{k, m-i}^{9}$ равенства (21) и его преемника в схеме $R$. Далее, в схеме $S_{k, m-i}^{9}$ переменная $x_{i 2}$ не поступает на вход элемента $\vee$ (см. рис. 7). Это значит, что при подстановке $x_{i-1,1}=0$ равенство (22) удаляется, то есть именно оно является преемником равенства (21) в схеме $R$. Отсюда вытекает, что $u_{i 2}=z_{i 1}^{\prime}$ и что при переходе от схемы $R$ к схеме $S_{k, m-i}^{9}$ переменная $z_{i 2}^{\prime}$ заменяется переменной $x_{i 2}$. Таким образом, равенство (22) принимает окончательный вид

$$
z_{i 2}^{\prime}=z_{i 1}^{\prime} \vee x_{i 2},
$$

где $z_{i 1}^{\prime}$ определяется равенством (21), и известно, что при обратном переходе от схемы $S_{k, m-i}^{9}$ к схеме $R$ переменная $x_{i 2}$ (см. рис. 7) заменяется переменной $z_{i 2}^{\prime}$.

Покажем, наконец, что $u_{i 1}=x_{i 1}$. Предположим, что это неверно. Представим схему $R$ (с той точностью, с которой мы выяснили ее строение) на рис. 8 .

Переменная $u_{i 1}$ не может совпадать с переменной $z_{i 0}$, так как в этом случае в схеме $R$ был бы цикл. Но тогда как переменная $x_{i 1}$, так и переменная $z_{i 0}$ поступает на вход только одного элемента и при подстановке $x_{i+1,1}=0$ (см. рис. 3 и 4) пропадает существенная зависимость выхода схемы $R$ от переменной $x_{i 1}$. Этого не происходит в схеме $S_{k, m-i+1}$, вычисляющей ту же функцию. Следовательно, $u_{i 1}=x_{i 1}$ и схема $R$ (рис.8) совпадает со схемой $S_{k, m-i+1}$ (рис. 4).

Таким образом, оказалось, что априори произвольная минимальная схема $R$ для функции $F_{k, m-i+1}$ определяется однозначно и совпадает со схемой $S_{k, m-i+1}$. Следовательно, схема $S_{k, m-i+1}$ является единственной минимальной схемой для функции $F_{k, m-i+1}$. Индуктивный переход завершен. Лемма доказана. 


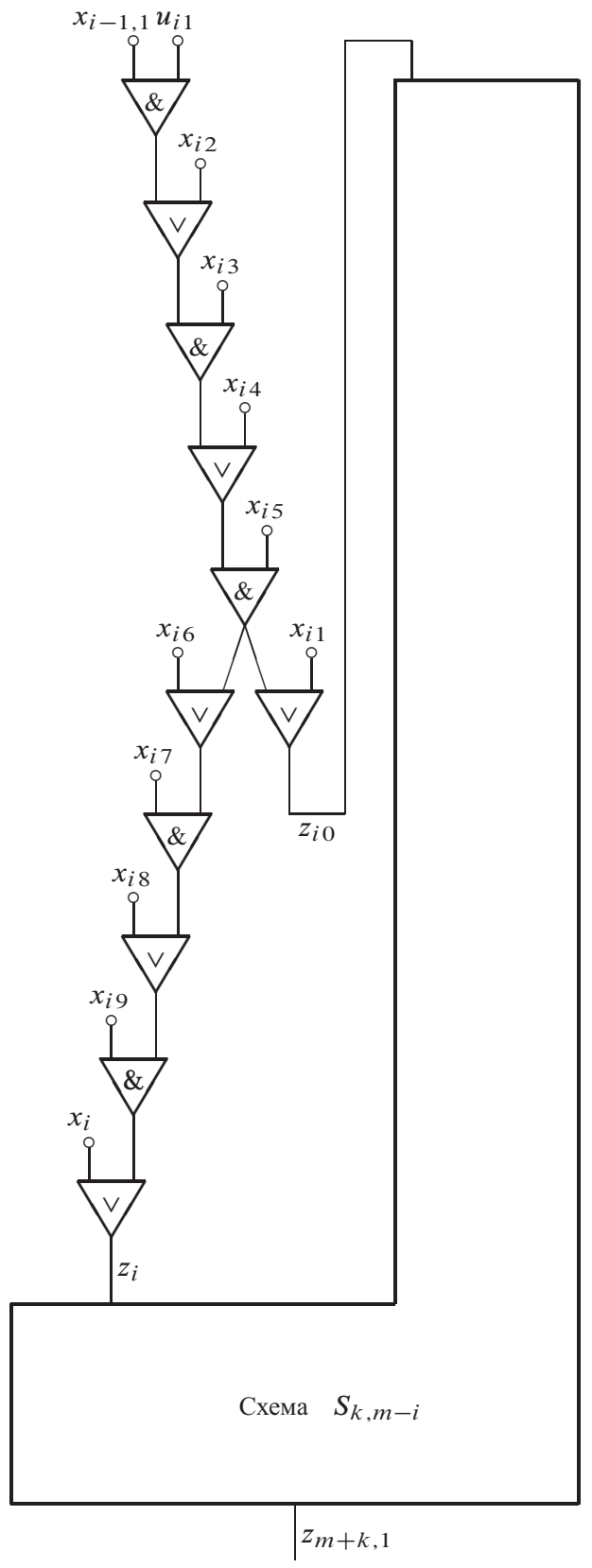

Рис. 8. Схема $R$ 
Прямым следствием лемм 1 и 4 является следующая теорема.

Теорема 1. Для каждой функции $F_{k}, k=1,2, \ldots$, существует единственная минимальная схема $S_{k}$ над базисом $B_{0}$, вычисляющиая эту функиию. При этом

$$
\begin{aligned}
& D\left(S_{k}\right)=6 \cdot 2^{k}+k+4, \\
& T\left(S_{k}\right) \leqslant k+16,
\end{aligned}
$$

и следовательно,

$$
T\left(S_{k}\right)<\log _{2} D\left(S_{k}\right)+14
$$

В заключение сделаем ряд небольших замечаний.

Замечание 1. Теорема 1 показывает, в частности, что в некоторых случаях нельзя уменьшить расхождение между глубиной и задержкой, переходя к другой минимальной схеме, вычисляющей ту же функцию.

А что произойдет, если ослабить условия перехода, допустив при переходе небольшое увеличение сложности схемы? Пусть $S-$ произвольная схема, для которой выполняется примерно такое же неравенство, как в теореме 1, то есть

$$
T(S)<\log _{2} D(S)+c,
$$

где $c$ - положительная константа. Тогда схему $S$ можно преобразовать в некоторую схему $S^{\prime}$ без ветвлений выходов элементов (то есть фактически в формулу), у которой

$$
D\left(S^{\prime}\right)=T\left(S^{\prime}\right)=T(S)
$$

(см. [4]). Оценив (ср. [1]) ее сложность

$$
\begin{aligned}
L\left(S^{\prime}\right) & <2^{D\left(S^{\prime}\right)} \leqslant 2^{T(S)}<2^{\log _{2} D(S)+c} \\
& =2^{c} D(S) \leqslant 2^{c} L(S),
\end{aligned}
$$

легко сделать вывод: если при эквивалентном преобразовании допускается усложнение схемы в $2^{c}$ раз, то расхождение между глубиной и задержкой можно устранить.

Скорее всего, это расхождение исчезает при значительно меньшем усложнении схемы, а само расхождение уменьшается очень быстро с увеличением допустимой сложности.

Замечание 2. В классе минимальных формул глубина всегда совпадает с задержкой, так как формулу, у которой глубина больше задержки, можно упростить, удаляя неработающие начальные участки некоторых путей (подробнее см. в [4]).

Замечание 3. В силу замечания 2 можно не различать понятия задержки и глубины, если речь идет о функции или системе функций.

В свое время работа была доложена на заседаниях семинара О. Б. Лупанова «Синтез управляющих систем и смежные вопросы» и получила поддержку. Светлой памяти моего учителя, замечательного ученого и человека Олега Борисовича Лупанова посвящаю эту статью. 


\section{Список литературы}

1. Храпченко В. М., Новые соотношения между глубиной и задержкой. Дискретная математика (1995) 7, №4, 77-85.

2. Лупанов О. Б., Об одном методе синтеза схем. Известия вузов. Радиофизика (1958) 1, №1, $120-140$.

3. Храпченко В. М., Нижние оценки сложности схем из функциональных элементов (обзор). Киберн. сб. (1984) 21, 3-54.

4. Храпченко В. М., Различие и сходство между задержкой и глубиной. Проблемы киберн. (1979) 35, 141-168.

Статья поступила 2.07.2006. 